\title{
EL CONCEPTO UEXKÜLLIANO DE MUNDO CIRCUNDANTE Y SUS DESPLAZAMIENTOS
}

\author{
Juan Manuel Heredia* \\ doi: 10.11144/Javeriana.uph38-76.cumc
}

\begin{abstract}
RESUMEN
Este artículo analiza el sentido que el concepto de mundo circundante (Umwelt) asume en Jakob von Uexküll, examina las distintas formulaciones que presenta en el desarrollo de su obra, y destaca las sucesivas redefiniciones que afectan a sus dos componentes: el mundo perceptible (Merkwelt) y el mundo de efectos (Wirkungswelt, Wirkwelt). Teniendo en cuenta el significativo impacto que el concepto de Umwelt tuvo en la filosofía continental, el propósito del estudio es reconstruir su sentido en función de las fuentes primarias y, a través de un análisis históricoconceptual, iluminar aspectos que no suelen ser tematizados en la literatura -en particular, aquellos relativos al mundo de efectos-.

Palabras clave: Jakob von Uexküll; mundo circundante; mundo perceptible; mundo de efectos; teoría del comportamiento
\end{abstract}

Universidad de Buenos Aires, Buenos Aires. Argentina. CONICET - Centro de Historia Intelectual (Universidad Nacional de Quilmes, Buenos Aires, Argentina).

Correo electrónico: jmheredia@filo.uba.ar

Para citar este artículo: Heredia, J. M. (2021). El concepto uexkülliano de mundo circundante y sus desplazamientos. Universitas Philosophica, 38(76), 15-47. ISSN 0120-5323, ISSN en línea 2346-2426. doi: 10.11144/Javeriana.uph38-76.cumc 


\title{
THE UEXKÜLLIAN CONCEPT OF SURROUNDING WORLD AND ITS DISPLACEMENTS
}

\begin{abstract}
This paper advances the analysis of the concept of Umwelt in Jakob von Uexküll's work, by examining its different formulations, and highlighting the successive redefinitions of its two components: the perception world (Merkwelt) and the effect world (Wirkungswelt, Wirkwelt). Considering the significant impact that the concept of Umwelt had on continental philosophy, the purpose of this study is to reconstruct its meaning through a historical-conceptual analysis, to illuminate some aspects that are not usually thematized in literature-in particular, those related to the effect world.
\end{abstract}

Keywords: Jakob von Uexküll; surrounding world; perception world; effect world; behavior theory 


\section{Introducción}

LA TEORÍA DE LOS MUNDOS CiRCUNDANTES que Jakob von Uexküll ${ }^{1}$ introduce en las primeras décadas del siglo XX, sus multifacéticas proyecciones y las derivas hermenéuticas a las que dio lugar configuran un capítulo destacado de la filosofía continental contemporánea. En efecto, este singular biólogo antidarwinista deja su huella en buena parte de los pensadores más destacados del siglo y, surcando distintas tradiciones teórico-metodológicas, se hace acreedor de no pocos elogios. Ortega y Gasset (1922/1965) declara que la teoría biológica de Uexküll ha ejercido "gran influencia" sobre él, y agrega: "no conozco sugestiones más eficaces que las de este pensador, para poner orden, serenidad y optimismo sobre el desarreglo del alma contemporánea” (p. 139). Heidegger (19291930/2007) señala que "sus investigaciones concretas son de lo más fructífero que la filosofía pueda apropiarse hoy" (p. 318), y dedica buena parte de su curso en Friburgo de 1929-1930 a reflexionar sobre las implicancias ontológicas de su teoría biológica. Cassirer (1950/1998, pp. 242-263) plantea que, metodológicamente, Uexküll contribuye decisivamente a superar la antinomia entre mecanicismo y vitalismo, y, por otro lado, se vale de su teoría de los mundos circundantes para repensar el sistema simbólico en que se emplaza el ser humano (Cassirer, $1944 / 2009$, p. 46). Uno de los fundadores de la teoría general de los sistemas, Ludwig von Bertalanffy (1968/1976), indica que el concepto uexkülliano de Umwelt inaugura "la actitud biológica moderna" (p. 239), abriendo paso a enfoques holísticos y organísmicos. Merleau-Ponty (1957-1958/1995) señala que,

1 Jakob von Uexküll nace en Estonia en 1864. Estudia zoología en la Universidad de Dorpat (hoy Tartu) y fisiología en la Universidad de Heidelberg, donde además se desempeña como asistente de Wilhelm Kühne. En estos años, alterna su trabajo con estancias de investigación en la Estación Zoológica de Nápoles, donde conoce a Hans Driesch y a otros biólogos de renombre. Publica su primer libro en 1905 y, desde entonces, no deja de participar del debate filosófico-biológico hasta su muerte en 1944. Construye una singular biología vitalista y es principalmente reconocido por sus aportes a la formación de la etología como subdisciplina. De hecho, en 1926, funda en Hamburgo el Institut für Umweltforschung (Instituto de Investigación Ambiental), al cual dirige hasta 1934 y desde donde potencia el fermento de la teoría del comportamiento animal. Sus principales libros son: Mundo circundante y mundo interior de los animales (1909), Ideas para una concepción biológica del mundo (1913), Biología teórica (1920), Teoría de la vida (1930), Andanzas por los mundos circundantes de los animales y los hombres (1934) y Teoría de la significación (1940). Para más información respecto del itinerario intelectual de Uexküll, véase Heredia, 2020. 
con dicho concepto, "Uexküll anticipa la noción de comportamiento" (p. 220) de la Gestaltpsychologie, y, por similares razones, Georges Canguilhem (1952/1971) destaca su "lucidez" al plantear filosóficamente el problema de la relación organismo-medio (p. 143). Deleuze y Guattari (1991/1993) se refieren a su biología teórica como una "obra maestra" (p. 188) y se valen de sus ideas para pensar las nociones de territorio, ritornelo y agenciamiento individuado. Peter Sloterdijk (2005) subraya el enorme impacto generado por el concepto uexkülliano de mundo circundante en la ecología y la filosofía del siglo XX, y plantea que la noción heideggeriana de ser-en-el-mundo "constituye una respuesta filosófica a la conmoción que le produjo [dicho] concepto biológico” (p. 945)². En la misma línea, Bruno Latour (2010/2012) lo presenta como "un pensador totalmente original” (p. 179) y recuerda que la noción de Umwelt, además de influenciar a los filósofos, constituyó una piedra angular para el desarrollo de la etología ${ }^{3}$. Este breve e incompleto repaso revela el interés que Uexküll ha despertado en la filosofía del último siglo, al tiempo que sugiere líneas de interpretación divergentes. De hecho, al calor de dichas lecturas y reapropiaciones del Umwelt uexkülliano, emergen distintos semblantes teóricos, desde el subjetivismo solipsista hasta el constructivismo posestructuralista, pasando por la fenomenología, el existencialismo y el neokantismo.

En el campo de los estudios especializados, sin embargo, hay una lectura claramente dominante, y es la que ha forjado la corriente biosemiótica al articular la semiótica de Pierce y la teoría de la significación de Uexküll (Kull et al., 2009). Iniciada en la década de 1980 a partir de los estudios de Thomas Sebeok y de Thure von Uexküll (hijo de Jakob von Uexküll), y profundizada luego por autores como Jesper Hoffmeyer, Claus Emmeche, Kalevi Kull, Terrence Deacon, y John Deely, la biosemiótica acredita actualmente numerosos desarrollos teórico-biológicos

2 "Among the first to respond to the provocation innate in the [Uexküllian] concept of the environment was Martin Heidegger, who as early as the mid-1920s grasped the ontological implications of the new biology. I would go so far as to say that his formulation of 'being-in-the-world' constitutes a philosophical response to the shock he felt when confronted by the biological concept of the environment" (Sloterdijk, 2005, p. 945).

3 Para un análisis atento de la relación de Uexküll con la etología de Konrad Lorenz, véase Brentari, 2009, 2015, pp. 217-224. 
y se ha establecido como el abordaje predominante en lo que respecta a la obra de Jakob von Uexküll (Sebeok, 2001; Thure von Uexküll, 2004; Castro García, 2009; Brentari, 2013, 2015; Guidetti, 2013; Kull, 2015, 2020; Araújo, 2016; Tønnessen et al., 2018). Por otro lado, hay que señalar que, en las últimas dos décadas, la importancia teórica e histórico-intelectual del biólogo estonio-alemán ha comenzado a ser reconocida y revalorizada también desde otros ángulos. $\mathrm{Al}$ hecho de que varios de sus libros han sido reeditados y/o traducidos en Alemania, Francia, Estados Unidos y Argentina, se suma la aparición de una serie de estudios dedicados íntegramente al análisis de su obra (Mildenberger, 2007; Buchanan, 2008; Castro García, 2009; Brentari, 2015; Michelini \& Köchy, 2020), así como investigaciones histórico-intelectuales e histórico-conceptuales que van más allá del enfoque biosemiótico (Harrington, 1996; Stella \& Kleisner, 2010; Chamois, 2016). Asimismo, una tercera línea de investigación en lo que respecta a Uexküll se manifiesta en los artículos que tematizan la recepción de sus conceptos en la filosofía occidental. En este punto, se registran ensayos sobre su impacto en Heidegger (Muñoz Pérez, 2015; Alsina Calvés, 2018; Michelini, 2020), Cassirer (Stjernfelt, 2011; Koutroufinis, 2016; Brentari, 2020), Ortega y Gasset (Acevedo Guerra, 1994; Utekhin, 2001; Olivé Pérez, 2018; Alonso Fernández, 2019), Husserl (Tønnessen et al., 2018, Moreno Mancipe, 2020), Merleau-Ponty (Ostachuk, 2013; Bacarlett Pérez, 2014; Moyle, 2020), Simondon (Arcila Rodríguez, 2018) y Deleuze (Hendlin, 2016; Cimatti, 2020).

Tanto en las reapropiaciones filosóficas como en la literatura especializada, la teoría de los mundos circundantes (Umwelten) suele ser el punto clave sobre el cual pivotean los análisis. $\mathrm{Al}$ respecto, y en términos esquemáticos, es posible identificar tres tipos de interpretaciones predominantes. Una lectura afirma que dicho concepto expresa una estructura a priori de carácter específico, esto es, una serie de esquemas trascendentales que condicionan positivamente la relación de cada especie animal con el mundo fenoménico. Dominique Lestel (2010) se refiere a dicha estructura como una "subjetividad de especie" (p. 8). Otra lectura, en polémica con aquella, relativiza la rigidez y el carácter específico de la instancia trascendental, y sostiene que el mundo circundante es producto de una subjetividad de tipo individual. Esta es la perspectiva que asume Camille Chamois (2016, pp. 183-190), quien, apoyándose en dos de los últimos libros de Uexküll, rechaza que el Umwelt pueda ser entendido como una estructura a priori de la 
especie, reivindica las dimensiones individuales y revaloriza el peso de las acciones basadas en la experiencia. Una tercera línea de interpretación defiende la idea según la cual el mundo circundante (Umwelt) no expresa la actividad de un sujeto constituyente (sea individual o específico), sino que es índice de un sistema subjetivo-objetivo. Esta es la posición del hijo del biólogo, Thure von Uexküll (2004), quien sostiene que la teoría de su padre "no es ni objetivista ni subjetivista, sino -como se describiría actualmente- 'sistémica', y que Uexküll “entendía el proceso vital como un sistema coherente en el que sujeto y objeto se definen como elementos interrelacionados en un todo mayor" (pp. 20-21) .

Nuestra intención es aportar una perspectiva histórico-conceptual a este campo problemático. Consideramos que, en el decurso de la obra uexkulliana, el concepto de mundo circundante asume distintos sentidos y es objeto de reformulaciones, manifestando centralmente un desplazamiento desde el problema de la percepción hacia el del comportamiento. Nuestra tesis es que, desde su introducción en 1909 hasta al menos 1913, dicho concepto asume un carácter netamente perceptivo (siendo sinónimo de la noción de mundo perceptible [Merkwelt]), mientras que a partir de 1920, y fundamentalmente en 1934, es posible advertir una mayor atención al aspecto operativo y, con ello, la dominante perceptiva se atenúa con una idea positiva de mundo de efectos o mundo efectual (Wirkungswelt, Wirkwelt). Creemos que este desplazamiento del centro de interés desde la percepción a la acción supone una redefinición sustancial del concepto de Umwelt, permite comprender algunas de las dispares interpretaciones a las que ha dado lugar, y resulta concomitante con una serie de transformaciones teóricas que expresan la evolución del pensamiento de Uexküll. Entre dichas transformaciones, acaecidas entre 1920 y 1934, cabe mencionar una progresiva revalorización de la dimensión individual por sobre la específica, una cada vez mayor tematización de "animales superiores" en detrimento de los "inferiores", una reivindicación de las acciones plásticas y basadas en la experiencia por sobre las acciones reflejas e instintivas, y la integración al análisis de nuevos

4 "A premissa epistemológica da teoria de Jakob von Uexküll não é nem objetivista nem subjetivista, mas - como se descreveria atualmente - 'sistêmica.' Isso quer dizer que ele entendia o processo vital como um sistema coerente em que sujeito e objeto se definem como elementos inter-relacionados em um todo maior." (Thure von Uexküll, 2004, pp. 2021). 
conceptos e investigaciones etológicas (como las de Konrad Lorenz a inicios de la década de 1930).

A efectos de fundamentar la tesis planteada, en lo sucesivo, analizaremos la génesis y el sentido del concepto de mundo circundante en correlación con el devenir de sus dos componentes principales: el mundo perceptible y el mundo de efectos (o mundo efectual). Estructuraremos la exposición en tres partes. En primer término, realizaremos un breve comentario respecto de la traducción al castellano de las nociones concernidas, así como del ingreso del pensamiento uexkülliano en el mundo de habla castellana. Luego, nos referiremos a la génesis del concepto de mundo circundante y analizaremos la noción de mundo perceptible. Por último, tematizaremos la revalorización del mundo de efectos y, tras analizar esta noción con cierto detalle, concluiremos explicitando el sentido de este artículo en el marco de los debates en torno al Umwelt.

\section{La recepción y traducción de Uexküll en el mundo de habla hispana}

El papel de Ortega y Gasset en la difusión del pensamiento de UeXKüLl en el mundo de habla hispana es ineludible, y explica en buena parte un hecho notable: la obra de Uexküll fue traducida al castellano antes, y en mayor medida, que al inglés, al francés o al italiano. Ya en 1922, producto del impulso del filósofo español (Acevedo Guerra, 1994, p. 137; Alonso Fernández, 2019, p. 57) sale a la luz por la editorial Calpe la primera edición de Ideas para una concepción biológica del mundo (Bausteine zu einer biologischen Weltanschaunng), publicado en Alemania en 1913. El volumen reúne una serie de artículos del período 19071913, en los cuales Uexküll expone las bases de su teoría biológica y analiza desde distintos ángulos el concepto de mundo circundante, introducido inicialmente en Umwelt und Innenwelt der Tiere (Mundo circundante y mundo interior de los animales) de 1909. Ideas para una concepción... se reedita en España en 1934 y da lugar, luego, a otras dos ediciones en Argentina por la editorial Espasa-Calpe $(1945,1951)$. Por otro lado, en marzo 1924, en el número 9 de la Revista de Occidente, se publica un artículo de Uexküll, "La biología de la ostra jacobea", 
por solicitud directa de Ortega, director de la revista. En 1925 se publica Cartas biológicas a una dama (Biologische Briefe an eine Dame, 1920), y en 1942 Meditaciones biológicas. La teoría de la significación (Bedeutungslehre, 1940), ambos por el sello editorial de la Revista de Occidente (que, por otra parte, publica en 1945 una segunda edición de Cartas biológicas... $)^{6}$. En 1944, por último, la editorial Summa de Madrid publica otro libro de Uexküll, Teoría de la vida (Die Lebenslehre, 1930). Lo que resulta notable en este punto es que casi ninguno de los libros mencionados ha sido traducido, aún hoy, al inglés o al francés. La única excepción la constituye La teoria de la significación, cuya traducción al inglés se publica recién en 1982, y en francés aparece en 1965 junto con Andanzas por los mundos circundantes de los animales y los hombres (Streifzüge durch die Umwelten von Tieren und Menschen, 1934), que había sido publicado por primera vez en inglés en 1957, y en italiano en $1967^{7}$. Este último libro fue traducido al castellano y publicado en 2016 por la editorial Cactus, la cual también publicó en 2014 una nueva traducción de Cartas biológicas...

Lo anterior no pretende ser un recuento exhaustivo, pero alcanza para destacar un hecho singular y documentado: fuera del ámbito germanoparlante, las ideas de Uexküll han fluido con mayor rapidez y en mayor cantidad en países de habla castellana que en otros. Y, a la luz del conjunto de los libros de Uexküll traducidos hasta el momento, esta afirmación sigue siendo válida aún hoy.

5 En un breve párrafo que precede al artículo, y que escribe el propio Ortega (en Uexküll, 1924), leemos: "von Uexküll es uno de los maestros mayores con que hoy cuenta la biología. Ha creado todo un sistema de ideas biológicas, obtenido en una serie de estudios, ejemplares por su minuciosidad y precisión. Le hemos pedido que desarrolle con motivo de un animal determinado sus puntos de vista generales, reuniendo así ambas facetas de su obra -el sistema y la investigación concreta-. Las páginas que nos envía son un admirable escorzo de su ideología biológica” (p. 297). Por otro lado, un hecho curioso es que en la publicación hay una confusión con el nombre del autor, quien aparece como "Jacques von Uexküll" cuando su verdadero nombre completo es Jakob Johann von Uexküll.

6 Cartas biológicas a una dama, asimismo, fue publicada en Chile por la Editorial Zig-Zag. La edición no consigna fecha de publicación, y no nos ha sido posible establecerla. No obstante, en la solapa de la portada del libro, se indica: "la presente edición [...] es la primera aparecida en español".

7 Para un informe preliminar de las traducciones y publicaciones de la obra uexkülliana fuera de Alemania, véase Kull, 2001, pp. 36-39. 
La cantidad de ediciones en castellano, sin embargo, encuentra como correlato un verdadero festival en lo que a la traducción de los términos refiere:

- El concepto menos conflictivo es el de Umwelt, traducido casi unánimemente como "mundo circundante" -excepto en la edición de Teoría de la vida de 1944, que no acredita al traductor, donde se traduce como "mundo visible"-.

- El concepto de Merkwelt, por su parte, ha sido traducido al castellano como "mundo perceptible" (R. M. Tenreiro; T. Bartoletti \& L. C. Nicolás); "mundo de la percepción” (M. García Morente; J. M. Sacristán; Revista de Occidente, 1924); "mundo de observación" y "mundo observable” (editorial Summa, 1944); y "mundo perceptual” (M. Guntin). En otras lenguas también encontramos una multiplicidad de versiones: en francés, aparece traducido como monde de laperception y mondeperceptif, y en inglés como perceptive world, world-as-perceived, perception world, perceptual world y world-as-sensed.

- En tercer lugar, en lo que respecta a los conceptos de Wirkwelt y Wirkungswelt, encontramos las siguientes alternativas castellanas: "mundo de efectos" (R. M. Tenreiro; T. Bartoletti \& L. C. Nicolás); "mundo de la acción" (M. García Morente); "mundo de la actuación" (Revista de Occidente, 1924); “mundo de acción” (J. M. Sacristán); "mundo operativo" (editorial Summa, 1944); y "mundo efectual” (M. Guntin). Las traducciones inglesas y francesas tampoco son unívocas: operative world, world-as-acted-upon, effect world, active world, operational world, world of action, monde d'action, monde actantiel.

Por detrás de esta multiplicidad terminológica es posible capturar algunas convergencias semánticas. En lo que respecta al Merkwelt, nos encontramos con el mundo que es perceptible para un sujeto animal, esto es, el mundo de $s u$ percepción. Esta noción se distingue explícitamente de la idea de mundo exterior (Außenwelt), entendida como la totalidad de estímulos materiales o energéticos que pueblan una determinada área de espacio geográfico. De esta totalidad, subraya Uexküll, el animal es capaz de recibir y procesar solo una pequeña parte en función de su estructura fisiológica y biológica - esto es, sus receptores, sus órganos centrales de percepción y sus esquemas-. Por otro lado, la noción de 
Wirkwelt -o Wirkungswelt, en su última versión- remite a la capacidad operativa y activa del animal, al hecho de que produce efectos en las entidades del mundo exterior y, con ello, se efectúa. Se podría hablar, por tanto, de un mundo de las efectuaciones. Wirkung remite al término latino effectus y Welt a mundus, mientras que el verbo alemán wirken supone en castellano los sentidos de producir un efecto, operar, actuar, obrar. Así como en el caso del mundo perceptible, el mundo de efectos y efectuaciones de un sujeto animal guarda una estrecha relación con su estructura fisiológica y biológica -efectores, órganos centrales efectores y secuencias de impulsos musculares-. En lo sucesivo, analizaremos ambos mundos en relación con el concepto base de Umwelt, destacando sus cambiantes sentidos en el desarrollo de la obra y, particularmente, la redefinición de las nociones de Wirkwelt y Wirkungswelt.

\section{Mundo circundante y mundo perceptible}

UEXKüLl NO ES EL PRIMERo EN EMPLEAR LA EXPRESión "MUNDO CiRCUNDANTE". Castro García (2009) indica que el origen del neologismo Umwelt corresponde a un poeta romántico, el danés Jens I. Baggesen, quien lo introduce en un pasaje de su libro Parthenaïs oder die Alpenreise (1803-1804). Stella y Kleisner (2010) también señalan dicha génesis y, en lo que respecta al sentido del concepto, plantean que su procedencia ya es rastreable en el Ensayo sobre el origen del lenguaje (1772), donde Herder habla de un nuevo "punto de vista" que podría contribuir a iluminar la psicología humana:

Este punto de vista es la esfera de los animales [Sphären der Tiere].

Todo animal posee un círculo al que pertenece desde el nacimiento, en el que ha entrado inmediatamente, en el que permanece a lo largo de su vida y en el que muere. Pero es curioso que, cuanto más agudos son sus sentidos y más admirables sus obras de arte, tanto más pequeño es su círculo, tanto más peculiar es su obra artística. [...] La araña teje con el arte de Minerva, pero todo su arte se halla igualmente entretejido en ese limitado espacio de tela; ese es su mundo. ¡Qué admirable es el insecto y qué estrecho su círculo de acción!

En cambio, cuanto más variadas son las funciones y la determinación de los animales, cuanto más inestable es su modo de vida, en una palabra, cuanto 
más amplia y variada es su esfera, tanto más vemos que su sensibilidad se divide y debilita. [...] Me es, por tanto, lícito asumir el siguiente principio: la sensibilidad, las habilidades y los instintos artísticos de los animales incrementan su fuerza e intensidad en proporción inversa a la magnitud y variedad de su círculo de acción (Herder, 2015, pp. 18-19).

No es en Herder ni en el romanticismo donde Uexküll encuentra patrocinio para su teoría, sino en Kant, al cual celebra y reivindica en toda su obra. Siguiendo la medular lectura que realiza Cassirer (1998, pp. 242-262), distintos autores, como Mildenberger (2007, p. 72) y Castro García (2009, pp. 67-86), han subrayado la importancia que la tercera crítica kantiana habría tenido en el biólogo estonio-alemán, señalando que ella le permite validar metodológicamente el empleo de juicios teleológicos frente al reduccionismo mecanicista. Otros autores han planteado que, más que la tercera, es la primera crítica la que lo hace trabajar: Harrington (1996) señala que, contra el positivismo neokantiano y el materialismo, Uexküll reivindica una lectura subjetivista e idealista de Kant (pp. 46-47); Stjernfelt (2011) indica que "mientras Cassirer es un neokantiano epistemologizante con fuertes tendencias objetivistas, Uexküll es un kantiano naturalista con fuertes tendencias subjetivistas" (p. 171) Crítica de la razón pura es el verdadero punto de partida filosófico para la teoría de los mundos circundantes, y no la Crítica del juicio.

Y, en efecto, la teoría uexkülliana se desarrolla en el marco de una profunda recuperación de la filosofía trascendental de Kant, la cual es repensada a la luz de la observación de la actividad de los animales en el espacio, y da lugar a la idea según la cual los animales no son máquinas mecánicas -como suponía la fisiología mecanicista-, sino sujetos que poseen formas de sensibilidad específicas, condiciones a priori de percepción, que determinan su inserción en el entorno geográfico (Umgebung) mediante la constitución de un mundo circundante (Umwelt). En función de su plan de construcción y de su estructura biológica (Bauplan), cada especie posee un determinado mundo perceptible (Merkwelt) $\mathrm{y}$, consecuentemente, cada animal específico es sensible solo a un conjunto

$8 \quad$ "We might schematically say that while Cassirer is an epistemologizing neo-Kantian with strong objectivist tendencies, Uexküll is a naturalizing Kantian with strong subjectivist leanings." 
limitado de elementos significativos, siéndole totalmente indiferente e inaccesible el resto del entorno y su multiplicidad de estímulos. De este modo, por detrás de la individualidad singular de cada animal, cabe reconocer un segundo orden de subjetividad: condiciones trascendentales específicas que prescriben qué puede y qué no puede percibir un animal. Uexküll introduce esta teoría en Umwelt und Innenwelt der Tiere (1909), donde recapitula sus investigaciones empíricas en el campo de la fisiología sensorial de los animales marinos, analiza los mundos perceptibles de las medusas, los erizos de mar y los pulpos, e introduce los conceptos de mundo circundante (Umwelt) y mundo interior (Innenwelt). Estas nociones suponen un nuevo punto de vista:

Solo una mirada superficial puede llevar a pensar que los animales marinos viven en un mundo uniforme, común a todos. Una mirada más atenta nos enseña que cada una de estas innumerables formas de vida diferentes posee su propio mundo circundante, el cual está en una relación de determinación recíproca con el plan de construcción del animal. [...] Si se investiga cuidadosamente esta conexión entre el plan de construcción y los factores externos, alrededor del animal se expande un mundo nuevo, completamente diferente del nuestro, su mundo circundante. (pp. 5-6)9.

Antes de avanzar con la noción de mundo circundante, cabe aclarar que Uexküll (1909, p. 5) rechaza tajantemente los abordajes psicológicos y subraya que su concepto de mundo interior es netamente fisiológico, y de ningún modo remite a algo así como un alma animal. Dicho mundo conceptualiza el funcionamiento del sistema nervioso central, el cual tiene lugar una vez que los receptores han sido afectados. En el mundo interior, Uexküll distingue un órgano perceptivo -que, conectado con los receptores, transforma los estímulos recibidos y transmite olas de excitación-, un órgano efector -conectado con los efectores que actúan sobre el mundo exterior- y un órgano central o conductor

9 „Nur dem oberflächlichen Blick mag es erscheinen, als lebten alle Seetiere in einer allen gemeinsamen gleichartigen Welt. Das nähere Studium lehrt uns, daß jede dieser tausendfach verschiedenen Lebensformen eine ihm eigentümliche Umwelt besitzt, die sich mit dem Bauplan des Tieres wechselseitig bedingt. [...] Ist dieser Zusammenhang des Bauplanes mit den äußeren Faktoren sorgsam erforscht, so rundet sich um jedes Tier eine neue Welt, gänzlich verschieden von der unsrigen, seine Umwelt." (Uexküll, 1909, pp. 5-6). 
-que procesa y hace circular los mensajes nerviosos y las olas de excitación entre los órganos perceptivo y efector-. El mundo interior traduce a un lenguaje de señales nerviosas los estímulos que afectan a los receptores y constituye, así, una red de conexiones nerviosas que no se confunde con los eventos y entidades objetivas del mundo exterior (Uexküll, 1909, p. 59). No obstante, más allá de esta noción fisiológica, lo que para Uexküll es fundamental es la dimensión teóricobiológica del problema, esto es, el hecho de que el animal no percibe estímulos cualesquiera, sino un conjunto limitado y definido de estos. La respuesta a este problema es el concepto de mundo perceptible: el animal solo percibe aquellos estímulos que son, para él, Merkmalträger -portadores de marcas perceptuales, portadores de características o, como los llamará en sus últimas obras, portadores de significación-. El objeto exterior que emite un estímulo significativo para un animal específico es, por tanto, portador de una marca perceptual, y el conjunto de marcas perceptuales (Merkmal) compone el mundo perceptible de la especie.

En un artículo publicado en 1910, Die Umwelt (El mundo circundante), Uexküll ahonda en las implicaciones filosóficas de dicha noción y plantea que, desde el punto de vista metodológico, la investigación debe identificar -mediante la observación experimental- ante qué cualidades de un objeto reacciona un animal para, luego, avanzar en la determinación de los contornos de su mundo perceptible y del sentido vital que asumen las marcas perceptuales identificadas (Brentari, 2015, p. 80). En Ideas para una concepción biológica del mundo (1913/1951), explicita la asociación estrecha que liga a las nociones de mundo circundante y mundo perceptible:

Para designar este mundo, que es el producto del organismo, he intentado introducir la palabra Umwelt (mundo circundante). La palabra se ha naturalizado prontamente, pero no la idea. Este término es empleado ahora para designar lo que rodea inmediatamente a un ser vivo, en el mismo sentido que antes la palabra milieu. De este modo ha perdido su sentido peculiar. Es un afán totalmente vano querer oponerse contra el uso del lenguaje, y tampoco la expresión "mundo circundante" corresponde con bastante exactitud al concepto que le es atribuido. Por ello quiero poner en su lugar el término "mundo perceptible", Merkwelt, y significar con ello que para cada animal hay un mundo especial, que se compone de las notas distintivas recogidas por él del mundo exterior. El mundo perceptible, Merkwelt, que solo depende de 
los órganos de los sentidos y del sistema nervioso central, se completa por el "mundo de efectos", Wirkungswelt, que abarca aquellos objetos a los cuales están acomodados los instrumentos de comer y moverse del animal. En el mundo de efectos se suele hoy pensar, ante todo, al hablar del mundo circundante como milieu de un animal [...]. Pero aquí reside el error fundamental. La enumeración de los diversos objetos del mundo general de efectos al cual está acomodado cada animal es de un interés totalmente accesorio y fácil de ejecutar por la pura observación. Pero el descubrimiento del mundo perceptible de cada animal es un trabajo principalmente nuevo y muy fatigoso, que solo puede ser resuelto mediante el experimento (Uexküll, 1951, p. 55).

En esta etapa de su pensamiento, la apropiación biológica de la filosofía trascendental kantiana, y la predominancia del mundo perceptible en el mundo circundante, conduce a Uexküll (1951, pp. 116-117) a presentar su teoría como una "biología subjetiva" que, a la zaga de una anatomía y una fisiología subjetivas, se contrapone a las tendencias objetivistas y fisicoquímicas que no ven más que fuerza, materia y movimiento en el mundo. De hecho, esta oposición se traslada al naciente concepto de mundo circundante: el mundo de efectos "es aquel que habitualmente llamamos mundo objetivo" y al mundo perceptible "lo designamos habitualmente como mundo subjetivo" (Uexküll, 1951, p. 160); "el mundo de efectos, común a todos (en el cual nos movemos nosotros y todos los animales), era al mismo tiempo la medida objetiva para todos los objetos, y se ha convertido ahora en nuestro mundo perceptible" (p. 150); "el mundo de las acciones y reacciones es el mundo de efectos" (p. 204]); "en el mundo de efectos no hay más que puras causas; en el mundo perceptible, relaciones” (p. 206). De lo dicho se desprende que la noción de "mundo de efectos" resulta, en este periodo, exterior a la teoría subjetiva uexkülliana y, por ello, se puede decir que "animales que viven en el mismo mundo de efectos poseen mundos perceptibles totalmente diversos" (p. 56). Uexküll (1951) rechaza así una visión spinozista del mundo material, según la cual un cuerpo en movimiento o en reposo remite siempre a otro cuerpo que lo determinó al movimiento o al reposo, y así sucesivamente, y sostiene en cambio que el animal es un sujeto autónomo, "un ser que convierte todos los valores ajenos en valores propios y crea entre esos valores propios relaciones que no poseen los valores exteriores" (p. 142). 
Llegados a este punto, se plantea un problema. ¿El mundo perceptible es constituido por el sujeto viviente o, más bien, es su condición? ¿El animal selecciona, interpreta y valoriza los signos del mundo exterior o, por el contrario, responde a un conjunto de señales predeterminadas que intermitentemente lo invaden desde fuera? Esta cuestión supone, por un lado, una distinción entre la especie y el individuo, y, por el otro, una diferenciación de grado entre las diversas especies. Respecto de lo primero, Uexküll sostiene que, si bien a nivel de la estructura específica el mundo perceptible está prefigurado a priori, y toda especie moviliza un segundo orden de subjetividad, a nivel del viviente singular hay una efectiva constitución subjetiva del mundo circundante. Esta se lleva a cabo "conforme a plan" (Planmäßigkeit) (es decir, en función de reglas inmanentes), pero es el individuo el que la efectúa concretamente en circunstancias dadas. Desde el punto de vista del individuo, por tanto, hay una determinación recíproca entre sujeto y mundo circundante. El viviente no se adapta por ensayo y error a un mundo exterior hostil; constituye su mundo resolviendo problemas. “¿Cómo es que los animales encuentran siempre la adecuada solución?”, se pregunta retóricamente Uexküll (1951, p. 36): "porque siempre les son presentados los debidos problemas”. El mundo perceptible delimita el campo de lo problemático y conduce indirectamente, a través del individuo, a la resolución.

Lo cual nos lleva al segundo punto. Mientras que el mundo perceptible de los animales simples se compone de un número muy limitado de marcas perceptuales -a cuyos estímulos y excitaciones responden de modo cuasi-inmediato-, los animales superiores acreditan un sistema nervioso más complejo que supone una mayor actividad de mediación. Es decir, poseen una mayor capacidad para procesar y articular los datos perceptibles y, diferenciando distintas redes receptoras, constituyen esquemas que les permiten percibir no ya estímulos o excitaciones simples, sino formas -esto es, colecciones de estímulos o series de excitaciones-. Los esquemas dan lugar a lo que Uexküll (1951) llama antimundo (Gegenwelt): "en este solo existe una selección de aquellas formas que son importantes para la vida del animal” (p. 187). Así, cuanto mayor sea el antimundo de un animal, más esquemas poblarán su órgano perceptivo, y más rico y variado será su mundo perceptible (p. 188). Esta diferencia, asimismo, se vincula con la distinción que Uexküll introduce entre animales de instinto y animales de experiencia: los primeros se distinguen de los segundos en que "nunca adquieren nada por 
experiencia, sino que su primera serie de acciones siempre sigue siendo la más perfecta" (Uexküll, 1951, p. 196).

De lo dicho se deriva que la diferencia entre las distintas especies se tematiza, en esta etapa del pensamiento uexkülliano (1909-1913), apelando a características fisiológicas que tienen sede en el mundo interior -la mayor o menor complejidad del sistema nervioso central- y, particularmente, a la relación entre los órganos perceptivos de dicho mundo y el mundo perceptible - esto es, a la función subjetiva en la constitución del mundo circundante-. Correlativamente, el órgano efector asume un rol totalmente subsidiario en la determinación de las acciones. "Hasta muy arriba en el mundo de los vertebrados", afirma Uexküll (1951), la especie de movimiento de los efectores está en alto grado determinada por una propia maquinaria nerviosa, que solo puede ser detenida o acelerada por la influencia de los órganos de los sentidos, pero no modificada. [El mundo perceptible] no contiene otra cosa que los signos para el comienzo de una acción, mientras que esta misma está predeterminada con todo detalle en el animal (p. 148).

En este marco, el mundo de efectos -como espacio descentrado y puramente causal- oficia como elemento negativo que permite diferenciar las distintas especies en función de sus facultades de percepción:

Mientras en los animales inferiores les competen los temas principales a los órganos efectóricos y su vida se desenvuelve principalmente en el mundo de efectos, en los animales superiores los órganos receptóricos representan los papeles directores, gracias al antimundo, con lo cual es atribuida al mundo perceptible una significación cada vez más grande. Y, finalmente, en el hombre el mundo perceptible vence por completo, en su desarrollo, al mundo de efectos, pues hasta sus propios efectores llegan a ser objetos de este mundo perceptible (Uexküll, 1951, p. 188).

La predominancia de los órganos perceptivos (o "receptóricos") por sobre los efectores, por último, se vincula a que en esta etapa Uexküll sigue apegado a la noción de reflejo para pensar las acciones (Uexküll, 1951, pp. 20 y ss.). Esta noción será eclipsada en estudios posteriores y abrirá la posibilidad de tematizar de otro modo la vida exterior de los animales. 


\section{Mundo circundante y mundo de efectos}

UEXKÜLL NO ABANDONARÁ SU INTUICIÓN ORIGINAL en lo que respecta a su teoría de los mundos circundantes, pero, en sus obras de 1920 y 1934, rectificará una serie de conceptos, abandonará otros y, en conjunto, matizará la centralidad del mundo perceptible en dirección a poder pensar con mayor precisión el mundo de efectos (Wirkungswelt, Wirkwelt). En este desplazamiento de la percepción a la acción, como veremos, convergen tres procesos: una mayor atención a los animales superiores en detrimento de los inferiores, un creciente interés por la vida exterior de los animales en desmedro de su mundo interior, y una tematización cada vez más atenta de la existencia individual por sobre la estructura de la especie. En este recorrido, los conceptos de reflejo y de antimundo pasarán a un segundo plano y, en su lugar, aparecerán nuevas nociones.

Como apunta Brentari (2015, pp. 97-98), en la segunda edición revisada y corregida de Umwelt und Innenwelt der Tiere (1921) el capítulo "El reflejo", incluido en la primera edición, es reemplazado por otro intitulado "Los círculos funcionales” (Der Funktionskreis). Con esta noción, Uexküll conceptualiza la vida exterior de los animales y el modo a través del cual los efectos de las acciones realizadas (o en curso de realización) repercuten en la percepción, la dirección y el control del comportamiento. Contra las ideas de causa eficiente, tropismo y reflejo, el círculo funcional presenta un modelo de causalidad retroactiva que articula dinámicamente el fijismo del mundo perceptible a priori y el constructivismo vital de los animales con sus efectuaciones a posteriori. De este modo, a través de las relaciones del sujeto animal con los objetos ${ }^{10}$, el mundo perceptible y el mundo de efectos se retroalimentan y condicionan recíprocamente,

10 Hay que tener en cuenta que, según Uexküll, los animales nunca se relacionan con objetos en sí, sino con determinadas cualidades o características de estos (solo aquellas marcas perceptuales que son capaces de percibir en función de sus estructuras fisiológicas y biológicas específicas). A la naturaleza intrínseca del objeto, inaccesible para el animal, Uexküll (1924) la denomina "contraestructura” (Gegenfüge) (p. 302). Asimismo, en Teoría de la significación (1940/1942), Uexküll plantea inequívocamente: “jamás un animal entra en relación con un 'objeto'. Merced únicamente a la relación, el objeto se transforma en portador de una significación que el sujeto le imprime” (p. 19). La aclaración es pertinente porque, a propósito del Umwelt uexkülliano, las lecturas de Scheler (1928/2003, p. 64) y Heidegger (1929-1930/2007, pp. 247 y ss.], subrayarán que, a diferencia de los animales, los seres humanos son capaces de relacionarse con objetos neutros y con el ente "en 
constituyendo perceptivamente y construyendo operativamente el mundo circundante. En este sentido, en Cartas biológicas a una dama (1920a/2014), Uexküll redefine los andamiajes de su teoría y plantea:

El mundo circundante se descompone en dos partes: en un mundoperceptible [Merkwelt], que va desde el portador de características hasta el órgano sensorial, y en un mundo de efectos [Wirkungswelt], que va desde el efector hasta el portador de características.

Hay un efecto [...] que va desde las características del portador de características hasta el órgano sensorial del animal. En el mundo interior, este efecto sufre diversos cambios y sale a la luz como una acción del animal para ponerse en funcionamiento en el portador de características. Así se cierra un círculo que denomino circulo funcional [Funktionskreis] y que siempre encierra al portador de características y al sujeto. La vida exterior de todo animal se compone de un número mayor o menor de círculos funcionales. El resultado del funcionamiento es siempre una destrucción o transformación del portador de características (2014, p. 88).

Uexküll señala que la existencia de todo animal se organiza en función de cuatro grandes círculos funcionales, dentro de los cuales hay, asimismo, numerosos círculos parciales que organizan cada una de las actividades. Dichas áreas fundamentales de funcionamiento, que suponen percepciones y acciones específicas, son: el medio natural de existencia (acuático, aéreo, etc.), la presa o el alimento, el enemigo o predador y la pareja sexual (Uexküll, 1920b/1926, pp. 127-129). Por otro lado, en Biología teórica (1920), el biólogo estonio-alemán distingue dos tipos de círculos funcionales. Mientras que el primero (figura 1) es un sistema unidireccional y se encuentra dominado por los esquemas a priori de la instancia trascendental (el mundo perceptible), el segundo (figura 2) es bidireccional y permite tematizar la formación de círculos de segundo grado, esto es, nuevas reglas perceptivo-activas, nuevas reglas de función, que, surgidas de la interacción práctica y sensoriomotora, pueblan con nuevos esquemas e impulsos el mundo

cuanto tal". Para Uexküll ni siquiera esto es cierto, pues no hay una diferencia de naturaleza sino de grado entre los mundos circundantes de los animales y los seres humanos. 
interior del animal. El segundo tipo refiere a la capacidad que acreditan los animales superiores y da lugar a nuevas nociones para pensar la acción en su especificidad. En este sentido, más allá de las acciones reflejas y las instintivas, Uexküll (1926, pp. 276-278) introduce las nociones de acción plástica (Plastische Handlung), acción basada en la experiencia (Erfahrungshandlung) y acción controlada (Kontrollierte Handlung). Mientras que la primera tematiza la formación de nuevos esquemas en el órgano perceptivo, y la segunda la creación de nuevas reglas de acción (secuencias de impulsos) en el órgano efector, la tercera conceptualiza una fluida comunicabilidad entre esquemas e impulsos, entre reglas de percepción y de acción, que se retroalimenta en el espesor del tiempo presente.

Figura 1

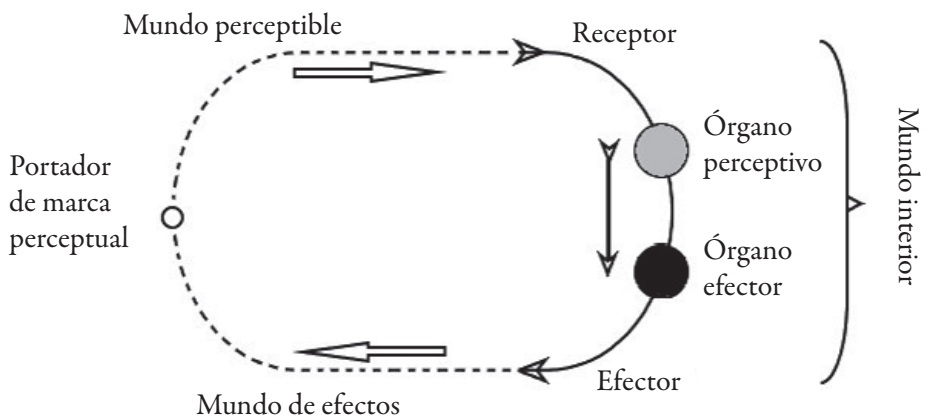

Fuente: Elaboración propia, con base en: Uexküll, J. J. (1920b).

Theoretische Biologie. Berlin: Verlag von Gebrüder Paetel, p. 155.

Figura 2

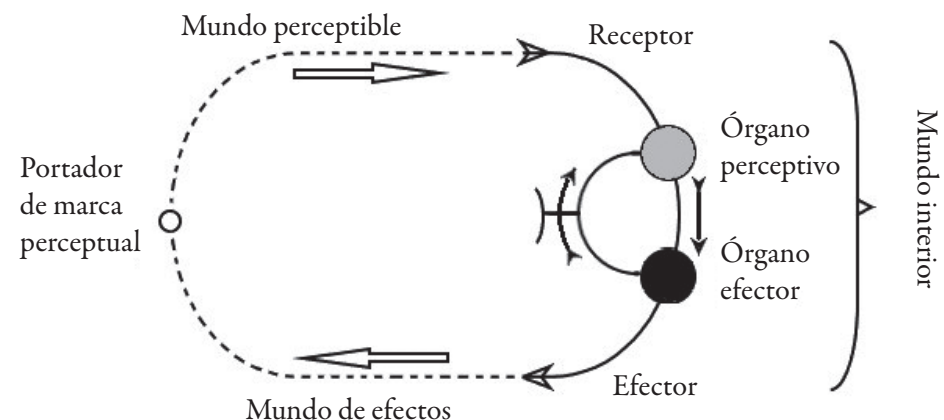

Fuente: Elaboración propia, con base en: Uexküll, J. J. (1920b).

Theoretische Biologie. Berlin: Verlag von Gebrüder Paetel, p. 157. 
Con esta batería de nociones, y sobre la base del segundo modelo de círculo funcional, Uexküll avanza con una teorización que va más allá de la dimensión perceptiva. De ello da cuenta otra de las innovaciones que introduce en sus obras de 1920, la noción de secuencia de impulsos (Impulsfolge). Esta tematiza la relación que, en el mundo interior, mantienen los órganos efectores y los efectores, y da lugar a la idea de reglas de acción (o, como también las llama, melodías de impulsos). Con ello, el biólogo estonio-alemán complementa la noción de esquemas de percepción, que en el anterior periodo de su pensamiento acaparaba toda la atención, y se abre camino para pensar las reglas directrices que regulan el funcionamiento de los músculos y el desempeño de los efectores. Por medio de estos desplazamientos conceptuales, Uexküll avanza en una suerte de temporalización de la instancia trascendental, matizando y relativizando el fijismo de las estructuras fisiológicas y biológicas específicas (Baupläne). En este sentido, en un notable pasaje de Teoría de la vida (1930/1944), y refiriéndose a los mamíferos, señala:

El plan de función de los organismos lleva en sí la posibilidad de modificar ampliamente la rígida estructura espacial con ayuda de sus factores inmateriales, es decir, de los esquemas y las melodías de impulsos. Para ello, como si dijéramos, sustituye constantemente por nuevos mecanismos aquellos que acaba de emplear. Verdad es que el plan anatómico de organización de los órganos de los sentidos permanece inmodificado, al igual que el de los músculos. Pero las posibilidades de combinación de los signos de observación conducen a la continua formación de nuevos esquemas, y los impulsos que rigen los músculos se convierten en melodías de impulsos continuamente nuevas. En este proceso surgen constantemente nuevos aparatos de observación con sus correspondientes aparatos de operación, de manera que una misma función unas veces es realizada de una manera y otras veces de otra (1944, pp. 115-116).

Esta relativización parcial de la estructura fisiológica y biológica a priori es válida para los animales superiores y, en particular, para su existencia singular pues, como buena parte de sus contemporáneos, Uexküll no cree en la lamarckiana herencia de los caracteres adquiridos y considera inadmisible que la estructura de una especie varíe en función de los avatares empíricos de sus representantes. No obstante, lo que cabe destacar en este punto es que la revalorización de la dimensión 
activa y operativa pone en primer plano la existencia de los individuos singulares, en detrimento de la tematización de las condiciones trascendentales de la especie, y en particular de las condiciones perceptivas, que dominan en la etapa previa.

Esta refocalización en los individuos se hace explícita en Andanzas por los mundos circundantes de los animales y los hombres (1934/2016), libro en el cual Uexküll se adentra como nunca en la dilucidación del mundo de efectos. Esta noción es compleja y, como ya sugerimos, cambia su sentido en el devenir de la obra. Síntoma de ello es que, mientras que en 1913 Uexküll (1951, p. 55) señala que el mundo de efectos es algo fácil de observar, en contraste con el mundo perceptible, en 1920 no duda en afirmar que "los procesos del mundo perceptible [Merkwelt $]$ resultan más comprensibles que los procesos del mundo de efectos [Wirkungswelt] de los animales" (2014, p. 146). Esta dificultad es afrontada en el libro de 1934 y, en retrospectiva, es posible advertir una trayectoria según la cual el mundo de efectos va perdiendo progresivamente el carácter descentrado que asumía en 1913; el mundo objetivo exterior y "común a todos", espacio de acciones y reacciones, dominio de meras relaciones causales, se vincula cada vez más al sujeto y, en el límite, da lugar a una subjetivación práctica del mundo circundante.

En Andanzas por los mundos circundantes..., Uexküll reconceptualiza el sistema sujeto-mundo circundante, depura sus conceptos y avanza en la introducción de una idea positiva del mundo de efectos. En este sentido, plantea: "todo lo que un sujeto percibe se torna su mundoperceptual, y todo su obrar se vuelve su mundo efectual [Wirkwelt]"; ambos mundos "conforman juntos una unidad cerrada: el mundo circundante" (Uexküll, 2016, p. 35). En este marco, "cada sujeto animal aprisiona su objeto con los dos brazos de una tenaza: un brazo perceptual y uno efectual. Con el primero, le asigna al objeto un signo perceptual [Merkmal], mientras que con el segundo le asigna un signo efectual [Wirkmal]" (p. 43). Con esto, las marcas o signos perceptuales componen el mundo perceptible, las marcas o signos efectuales componen el mundo de efectos, y ambos grupos de signos son modos de subjetivar los objetos exteriores con los que se entabla una relación funcional.

Esta proyección de significados, que es exterior al cuerpo del animal, constituye/construye el mundo circundante (Umwelt) y supone una serie de procesos inmanentes al sistema nervioso central. Aquí, Uexküll distingue dos niveles, uno citológico y otro fisiológico, a los cuales se añade luego un tercer nivel, propiamente biológico, que estructura el Umwelt -esto es, los signos perceptuales y 
efectuales que el sujeto atribuye a los objetos-. En primer lugar, tras señalar que "cada célula viva es un maquinista que percibe y obra" (2016, p. 41), Uexküll plantea que las células cerebrales se dividen en células perceptuales (Merkzellen) y en células efectuales (Wirkzellen) o "células de impulsos". Ambos grupos de células, en segundo lugar, se agrupan y articulan en nexos que integran, bajo la forma de señales y sensaciones, a los órganos perceptual (Merkorgan) y efectual (Wirkorgan). Estos órganos centrales, por tanto, se componen respectivamente de una multiplicidad de señales perceptuales (Merkzeichen) y de señales efectuales (Wirkzeichen) correlativas, o bien de estímulos externos que se presentan como preguntas, o bien de impulsos musculares o secuencias de impulsos "portadores de las respuestas" (2016, p. 42).

Ahora bien, la multiplicidad de señales que pueblan los órganos perceptual y efectual no se unifican a sí mismas de modo inmanente y fisiológico, sino que conquistan una unidad de orden superior "por fuera del cuerpo animal, en unidades que devienen las propiedades de los objetos externos al sujeto animal" (Uexküll, 2016, p. 42). He aquí el nivel propiamente biológico, el mundo circundante. La relación del sujeto con un objeto exterior da lugar, por un lado, a marcas o signos perceptuales (Merkmal) y, por el otro, a marcas o signos efectuales (Wirkmal). Ambos órdenes de signos, a su vez, se articulan a través de círculos funcionales y atribuyen a un mismo objeto un sentido perceptivo y otro operativo. En este proceso interior-exterior, por último, "las propiedades que caen bajo el signo efectual necesariamente influyen sobre las propiedades portadoras del signo perceptual a través de todo el objeto, operando sobre este signo un efecto transformador" (Uexküll, 2016, p. 43).

De lo dicho se desprende que el mundo de efectos no es un espacio descentrado, homogéneo y neutro, sino que siempre encuentra su centro en un sujeto, el cual lo configura a partir de sus efectuaciones y acciones. En este sentido, dicho mundo se encuentra en paridad con el mundo perceptible, y no subordinado a este.

Ahora bien, más concretamente, ¿qué quiere decir que todo el obrar de un sujeto se vuelve su mundo de efectos? ¿Cómo pensar la especificidad de este mundo? El biólogo estonio-alemán no desarrolla lo suficiente esta cuestión, y dista de ofrecer una teoría articulada del mundo de efectos, pero en su libro de 1934 ofrece algunos elementos. El primero es el espacio efectual (Wirkraum). Se trata de una noción fisiológica que conceptualiza la relación entre las sensaciones 
del movimiento y el espacio tridimensional en que se desenvuelven. La unidad mínima de dichas sensaciones son las "señales direccionales" que, distribuidas en tres pares de planos direccionales (adelante/atrás, arriba/abajo, derecha/izquierda), tienen asiento en un órgano (el oído interno) dotado de "canales semicirculares". Estos se relacionan de modo inmanente con las tres dimensiones del espacio y dan cuenta de la existencia de un espacio efectual. Este último no es una multiplicidad caótica de señales direccionales, ni un espacio motriz homogéneo, sino que organiza los tres planos direccionales en un sistema de coordenadas cuyo centro es el cuerpo del sujeto. Lo interesante en este punto es que hay animales que, con total independencia de señales perceptuales, en ausencia de toda percepción visual, son capaces de orientarse en el espacio y definir el sentido de sus movimientos en función de un mapa efectual inmanente. Estos animales, dice Uexküll (2016, p. 57), poseen en sus canales semicirculares una suerte de "brújula apuntando a la propia 'puerta de casa". Y esto no solo se da en animales con hogar o hábitat fijo, sino también en insectos y moluscos. Para explicar esta idea, Uexküll (2016) evoca un experimento: "desplácese un panal de abejas dos metros mientras que la mayoría de las abejas se encuentren fuera. Se verá entonces a las abejas concentrarse al aire libre, allí donde solía estar el orificio de salida -su puerta de casa-. Solo una vez transcurridos cinco minutos podrán las abejas ajustar su orientación y volar hacia el panal” (pp. 57-58).

El segundo elemento asociado al mundo de efectos es el concepto de imagen efectual (Wirkbild). Al introducirlo, Uexküll lo contrapone al instinto y, más en general, a la teleología. Salvo raras excepciones entre los superiores, los animales no se conducen conforme a un fin, ni persiguen objetivos. $Y$ su comportamiento tampoco se explica apelando a una oscura y confusa idea de instinto. Los animales, según Uexküll, perciben y obran en función de un plan supraindividual, esto es, de un sistema de relaciones de significación que rige las relaciones entre heterogéneos, y de una estructura fisiológica y biológica (Bauplan) que condiciona al sujeto como unidad funcional. La imagen efectual tematiza, centralmente, cómo los animales estructuran funcionalmente el mundo a través de sus relaciones prácticas. Se trata de un discernimiento esencialmente operativo de los objetos, y no perceptivo. Para explicar esto, Uexküll evoca el caso del cangrejo ermitaño que, frente a la misma imagen perceptual de una anémona de mar, puede adoptar tres comportamientos distintos. Así, según su estado de ánimo 
(Stimmung), la anémona puede adquirir para el cangrejo significaciones prácticas o "tonos efectuales" distintos: tono defensivo, habitacional o alimenticio. De este experimento, Uexküll (2016) extrae la siguiente conclusión: incluso en el mundo circundante de los antrópodos, "la imagen perceptual proporcionada por los órganos sensoriales puede ser suplementada y alterada por una 'imagen efectual' dependiente de la acción que produce" (p. 103). Como hemos visto, todo objeto se le presenta al sujeto según signos perceptuales definidos, pero, en tanto el animal entra en trato con el objeto, le imprime un sentido práctico, un signo efectual.

En la "imagen efectual", por tanto, hay una predominancia de lo operativo por sobre lo perceptivo y, por otro lado, esa predominancia -que asigna tonos, poderes y cualidades funcionales a los objetos- supone en su institución un cierto coeficiente afectivo. Sea en humanos o en cangrejos, "el estado anímico del sujeto será determinante respecto de qué imagen efectual ha de prestarle cuál tono a la imagen perceptual" (Uexküll, 2016, p. 105). A esta impronta afectiva, propia de la imagen efectual, Uexküll también la llama "tono de búsqueda" (2016, p. 136). Y agrega que, en función de cómo dichos tonos impulsen al sujeto, una misma imagen perceptual puede asumir distintos significados funcionales en el mundo de efectos. En este sentido, los tonos de búsqueda son primeros con respecto a la imagen perceptual de lo buscado: "el sapo hambriento sale inicialmente a buscar comida apenas munido de un tono de alimentación genérico. Es solo después de haber devorado una lombriz o una araña que adquiere una imagen de búsqueda más específica" (Uexküll, 2016, p. 136).

Una última característica de las imágenes efectuales es la seguridad y la certidumbre que producen. $\mathrm{Al}$ respecto, y de modo similar al principio subrayado por Herder, el biólogo estonio-alemán señala:

El mundo circundante recién adquiere su admirable certidumbre para los animales que lo habitan al tomarse en cuenta los tonos efectuales. Podemos decir que un animal podrá distinguir tantos objetos dentro de su mundo circundante como actividades pueda efectuar. Si posee pocas imágenes efectuales como resultado de pocas actividades, su mundo circundante consistirá de pocos objetos. Como resultado de ello, el mundo podrá haberse empobrecido, pero también se habrá vuelto tanto más seguro. Porque es mucho más sencillo orientarse entre unos pocos objetos que entre una multitud (Uexküll, 2016, p. 106). 
El mundo de efectos, por último, encuentra elementos en los conceptos de senda familiar (bekannte Weg), hogar (Heim) y territorio (Heimat). El primer concepto tematiza fenómenos en los cuales, producto de experiencias recurrentes, el sujeto animal es capaz de guiarse y desplazarse en el espacio efectual sin recurrir a referencias dadas por la percepción. Es como si el animal interiorizase y diseñase un mapa efectual del mundo exterior, un mundo de efectos propio y no-perceptivo, hecho de direcciones, planos, impulsos, signos efectuales y tonos de búsqueda. ¿Cómo sucede esto en el sujeto? La repetición de señales efectuales se graba en la memoria, produce la fijación de una secuencia o melodía de impulsos en el sistema nervioso, y estructura un camino definido en función de un conjunto de señales direccionales inmanentes. Si bien en su génesis la senda familiar se vale de elementos perceptuales, una vez asentada por la experiencia, estructura un mundo de efectos propio, permitiendo al sujeto orientarse operativamente sin apelar a percepciones o estímulos exteriores. Así, una vez instituida, la senda familiar "funciona como un corredor de líquido ligero dentro de una masa viscosa" (Uexküll, 2016, p. 114). La experiencia sensorial no juega ningún rol en el caso de la "senda innata", en la cual el animal posee reglas de acción y secuencias de impulsos a priori que son totalmente independientes de la percepción. Uexküll (2016, p. 141) pone el ejemplo de la larva del gorgojo que, sin señal perceptiva alguna, y con una previsión asombrosa, cava un túnel en la arveja aún tierna a efectos de poder escapar cuando sea un gorgojo adulto. Años antes, el biólogo estonio-alemán se refería a este tipo de comportamientos como "acciones instintivas" pero, en el libro de 1934, el concepto de instinto no es solo abandonado, sino también repudiado. La idea de senda innata, por último, explicaría el fenómeno de las aves migratorias que, en función de una "ruta innata", son capaces de orientarse en un espacio inmenso y viajar de un continente a otro.

Las ideas de hogar y de territorio se asemejan en sus fundamentos a la senda familiar (Uexküll, 2016, p. 137). El territorio se presenta como un perímetro variable que, en general, rodea al hogar (nido, guarida, madriguera, casa). El espacio dentro de esas fronteras es parte del mundo de efectos del sujeto, es como una prolongación de su cuerpo, es su territorio. En él predominan los signos efectuales y los tonos que remiten al ataque y la defensa. El biólogo estonio-alemán ejemplifica el agresivo velar por los límites del territorio apelando a peces, urracas, arañas, topos, y subraya que la constitución del territorio es "un producto 
exclusivamente subjetivo. Su presencia jamás podría colegirse ni del más minucioso conocimiento del entorno" (Uexküll, 2016, p. 116).

Se plantea, por último, el problema de la temporalidad de los mundos circundantes. Para Uexküll (2016), "sin un sujeto viviente, no puede haber ni espacio ni tiempo" (p. 49), y ello quiere decir que la relación entre espacio y tiempo es intrínseca, que cada viviente habita en su propia esfera, que "cada Umwelt tiene sus propias dimensiones espaciales y temporales" (Uexküll, 1937/2001, p. 117). Uexküll (2016) recurre a la teoría de las "longitudes de momentos" de Karl Ernst von Baer, a quien, dice, "le corresponde el mérito de haber mostrado que el tiempo es el artificio de un sujeto" (p. 73). Esquemáticamente, la tesis es la siguiente: la vida de todo animal se compone de la misma cantidad de momentos, pero en cada especie la duración de los momentos es diferente. ¿Qué son los momentos?: "son los más pequeños recipientes indivisibles de tiempo", "son la expresión de sensaciones elementales indivisibles, las llamadas señales de momento" y, más importante, "el momento es el mismo para todas las áreas sensoriales, porque todas las sensaciones están acompañadas por la misma señal de momento [Merkzeit]" (Uexküll, 2016, p. 73). Imbuido en esta idea, Uexküll (2014) señala fascinado:

Ahora bien, hay animales que viven solo un año y otros apenas unos días. ¿Cómo se transforma la imagen del mundo si ellos alojan en su vida la misma cantidad de momentos que nosotros? [...] Los animales que viven solo un día les hablarían a sus hijos de ese tiempo horroroso como una vieja leyenda. Para algunos, día y noche serían un mes; para otros, media vida. A estos seres vivos, todos los sucesos en el mundo les deberían parecer extraordinariamente largos. Así, la bala que sale volando de la pistola parecería estar suspendida en el aire. Desconocerían también el crecimiento de los árboles, al igual que nosotros el de las montañas. Por otro lado, podemos pensar en seres vivos que extienden su cantidad de momentos durante un mayor número de años. Para ellos, las estaciones cambian como para nosotros los días. Todo ocurriría en un tiempo más acelerado. Las hierbas brotarían de la tierra como de una fuente y desaparecerían. Los bosques reverdecerían, crecerían y morirían como las praderas. No se vería el sol, solo un haz centelleante aparecería brevemente en el cielo para darle lugar a una corta oscuridad (p. 62). 
Lo dicho implica que, en función de las distintas longitudes de momentos, y conforme a las diversas temporalizaciones específicas, en los mundos circundantes de los animales, "los procesos motrices se desplieguen con mayor celeridad o lentitud que el nuestro" (Uexküll, 2016, p. 74). Se toma conciencia así de que cada uno de los innumerables mundos circundantes que hay a nuestro alrededor prolonga una duración cualitativa específica. Cada uno moviliza y representa un ritmo del tiempo que se enreda con otros ritmos y, en un mismo orden de coexistencias, se cruzan velocidades de vida absolutamente heterogéneas.

\section{Conclusión}

EN EL MARCo DE LA FRECUENTE RECUPERACión del concepto de mundo circundante (Umwelt) en diversas expresiones de la filosofía contemporánea, y considerando las distintas interpretaciones a las que ha dado lugar en la literatura especializada, este artículo se ha propuesto llamar la atención sobre un desplazamiento inmanente a dicho concepto y, correlativamente, ha buscado destacar algunos aspectos específicos de dos de sus componentes (el mundo perceptible y el mundo de efectos). Creemos que el análisis de estos últimos, y en particular del mundo de efectos (o mundo efectual), nos ha permitido situar y explicitar una serie de tensiones teóricas, así como alumbrar una tesis histórico-intelectual relativa a la evolución del pensamiento uexkülliano. En este sentido, y para concluir, cabe recapitular y puntualizar algunos elementos significativos que se desprenden del análisis.

En primer lugar, y conforme al recorrido efectuado, es posible afirmar que el concepto de mundo circundante es objeto de distintas formulaciones y redefiniciones en el devenir de la obra de Uexküll. Concretamente, consideramos que el desplazamiento del centro de interés desde el problema restringido de la percepción hacia una comprensión integral del comportamiento animal redefine la naturaleza del concepto de Umwelt, y permite advertir una evolución desde un enfoque centrado en la especie a una tematización cada vez más atenta de las diferencias individuales. Esta modificación, desplegada a partir de la década de 1920 y consumada en 1934, se encuentra apuntalada por una serie de procesos correlativos: una profundización del análisis del comportamiento de los "animales superiores" por sobre los "inferiores"; el desarrollo de un concepto de 
causalidad retroactiva (el círculo funcional) -y, con él, el despliegue de una teoría de las acciones que va más allá de las acciones reflejas y las instintivas, e instala conceptos para pensar acciones plásticas, acciones basadas en la experiencia y acciones controladas-; y una integración en el enfoque de Uexküll de los resultados de las investigaciones empíricas desarrolladas en el marco del Instituto de Investigación Ambiental (Institut für Umweltforschung), centro de investigación que funda en 1926 y dirige hasta 1934.

Dichos procesos, en segundo lugar, propician una redefinición del concepto de mundo circundante y encuentran un índice teórico en el cambio de estatuto que afecta al mundo de efectos. Esta noción no suele ser abordada en su especificidad, y este artículo ha buscado ponerla en primer plano y revalorizarla frente a las dimensiones perceptivas, semióticas y semiológicas que suelen predominar en los análisis relativos al Umwelt. Según nuestro análisis, con la evolución del pensamiento uexkülliano, dicha noción padece una transformación: deja de ser un espacio neutro y descentrado de puras relaciones causales, y se convierte en el teatro de operaciones de cada animal, en un mundo práctico subjetivado. Dicho en otros términos, el mundo de efectos, que en un primer momento aparece como un continente común para una heterogeneidad de mundos perceptibles coexistentes, se pliega y sedimenta sobre el animal, deviene una dimensión operatoria que le es propia y permite tematizar, dentro del mundo circundante, una dimensión no-perceptiva en el comportamiento. En este sentido, hemos buscado mostrar que las nociones de territorio, espacio efectual, imagen efectual, tono de búsqueda y senda familiar e innata apuntan a enriquecer la idea de mundo de efectos y a pensarla en su especificidad frente a la de mundo perceptible.

Por último, y en el marco de los debates que la literatura especializada mantiene a propósito del concepto de mundo circundante, creemos que la tesis históricoconceptual planteada ofrece elementos para repensar una tensión inmanente al concepto, oscilación que contrapone un segundo orden de subjetividad de carácter específico y un primer orden de subjetividad signado por diferencias individuales. Consideramos que este problema adquiere nuevos matices desde una perspectiva atenta a la evolución del pensamiento de Uexküll, contribuyendo a resituar el debate del Umwelt como "subjetividad de especie". Respecto de las lecturas que interpretan el mundo circundante en términos de sistema y relativizan el rol del sujeto en su constitución, nuestra percepción es que se focalizan casi exclusivamente en el 
último libro de Uexküll (Teoría de la significación, 1940/1942), donde el problema no es la relación entre el sujeto animal y su mundo circundante, sino la relación entre mundos circundantes heterogéneos. Entendemos que esta corriente de interpretación, dominante en la biosemiótica y en las lecturas posestructuralistas de Uexküll, es fructífera y productiva, pero paga el precio de poner entre paréntesis no pocos pasajes de la obra del biólogo, donde la reivindicación de un concepto fuerte de subjetividad es ineludible.

\section{Referencias}

Acevedo Guerra, J. (1994). La sociedad como proyecto en la perspectiva de Ortega. Santiago de Chile: Editorial Universitaria.

Alonso Fernández, M. (2019). Razón vital como bio-logía. La filosofía de Ortega y su relación con la ciencia biológica. Ludus Vitalis, 27(51), 43-66.

Alsina Calvés, J. (2018). La biología en Los conceptos fundamentales de la metafísica de Martin Heidegger: influencias de Hans Driesch y de Jakob von Uexküll. Filosofia e História da Biologia, 13(1), 61-70.

Arcila Rodríguez, S. (2018). Mundos animales: tejidos de afectos, signos y movimientos. El Astrolabio, 17(2), 16-28.

Araújo, A. (2016). Structure, Sign and Uexküll's Theory of Meaning: A Philosophical Approximation. Cognitio, 17(1), 13-38.

Bacarlett Pérez, M. L. (2014). Uexküll y Merleau-Ponty: una ontología del entre. Metatheoria, 5(1), 35-47.

von Bertalanffy, L. (1976). Teoría general de los sistemas. (Trad. J. Almela). México: Fondo de Cultura Económica.

Brentari, C. (2009). Konrad Lorenz's Epistemological Criticism towards Jakob von Uexküll. Sign Systems Studies, 37(3/4), 637-662. https://doi. org/10.12697/SSS.2009.37.3-4.13

Brentari, C. (2013). How to Make Worlds with Signs. Some Remarks on Jakob von Uexküll's Umwelt Theory. Rivista Italiana di Filosofia del Linguaggio, 7(2), pp. 8-21. https://www.doi.org/10.4396/20130701

Brentari, C. (2015). Jakob von Uexküll - The Discovery of the Umwelt between Biosemiotics and Theoretical Biology. Dordrecht: Springer. 
Brentari, C. (2020). Ernst Cassirer's Reading of Jakob von Uexküll: Between Natural Teleology and Anthropology. En: F. Michelini \& K. Köchy (Eds.), Jakob von Uexküll and Philosophy (pp.106-121). Oxford-New York: Routledge.

Buchanan, B. (2008). Onto-Ethologies. The Animal Environments of Uexküll, Heidegger, Merleau-Ponty and Deleuze. Albany: State University of New York Press.

Canguilhem, G. (1971). La connaissance de la vie. París: Vrin.

Cassirer, E. (1998). El problema del conocimiento (Vol. IV). (Trad. W. Roces). México: Fondo de Cultura Económica.

Cassirer, E. (2009). Antropología filosófica. (Trad. E. Ímaz). México: Fondo de Cultura Económica.

Castro García, O. (2009). El concepto de Umwelt y el origen de la biosemiótica (Tesis doctoral inédita). Universidad de Barcelona, Barcelona, España.

Chamois, C. (2016). Les enjeux epistemologiques de la notion d'Umwelt chez Jakob von Uexküll. Tétralogiques, 21, 171-194.

Cimatti, F. (2020). From Ontology to Ethology: Uexküll and Deleuze \& Guattari. En: F. Michelini \& K. Köchy (Eds.), Jakob von Uexküll and Philosophy (pp. 172-187). Oxford-New York: Routledge.

Deleuze, G., \& Guattari, F. (1993). ¿Qué es la filosofía? (Trad. T. Kauf). Barcelona: Anagrama.

Guidetti, L. (2013). Jakob von Uexküll tra Kant e Leibniz. Dalla filosofia trascendentale alla topologia del viviente. Rivista Italiana di Filosofia del Linguaggio, 7(2), 66-83. https://www.doi.org/10.4396/20130705

Harrington, A. (1996). Reenchanted Science: Holism in German Culture from Wilhelm II to Hitler. Princeton: Princeton University Press.

Heidegger, M. (2007). Conceptos fundamentales de metafísica. Mundo, finitud, soledad (1929-1930). (Trad. J. A. Ciria Cosculluela). Madrid: Alianza.

Hendlin, Y. (2016). Multiplicity and Welt. Sign Systems Studies, 44(1/2), 94110. https://www.doi.org/10.12697/SSS.2016.44.1-2.06

Herder, J. G. (2015). Ensayo sobre el origen del lenguaje. En: Herder (1-105). (Trad. P. Ribas). Madrid: Gredos. 
Heredia, J. M. (2020). Jakob von Uexküll, an intellectual history. En: F. Michelini \& K. Köchy (Eds.), Jakob von Uexküll and Philosophy (pp. 17-35). Oxford-New York: Routledge.

Koutroufinis, S. (2016). Animal and Human "Umwelt" (Meaningful Environment). Continuities and Discontinuities. Balkan Journal of Philosophy, 8(1), 49-54. https://doi.org/10.5840/bjp2016815

Kull, K. (2001). Jakob von Uexküll: An Introduction. Semiotica, 134(1/4), pp. 1-59. https://doi.org/10.1515/semi.2001.013

Kull, K. (2015). Introduction to Biosemiotics. En: P. Pericles Trifonas (Ed.), International Handbook of Semiotics (pp. 521-533). Dordrecht: Springer.

Kull, K. (2020). Jakob von Uexküll and the Study of Primary Meaning-Making. En: F. Michelini \& K. Köchy (Eds.), Jakob von Uexküll and Philosophy (pp. 220-237). Oxford-New York: Routledge.

Kull, K., Deacon, T., Emmeche, C., Hoffmeyer, J., \& Stjernfelt, F. (2009). Theses on Biosemiotics: Prolegomena to a Theoretical Biology. Biological Theory, 4, 167-173. https://doi.org/10.1162/biot.2009.4.2.167

Latour, B. (2012). Cogitamus. Seis cartas sobre humanidades cientificas. (Trad. A. Bixio). Buenos Aires: Paidós.

Lestel, D. (2010). Prefacio. De Jakob von Uexküll à la biosémiotique. En: J. J. von Uexküll, Milieu animal et milieu humain (pp. 7-23). París: Payot \& Rivages.

Merleau-Ponty, M. (1995). La Nature. Notes. Cours du Collège de France. París: Seuil.

Michelini, F. (2020). The Philosoher's Boredom and the Lizard's Sun: Martin Heidegger's Interpretation of Jakob von Uexküll's Umwelt Theory. En: F. Michelini \& K. Köchy (Eds.), Jakob von Uexküll and Philosophy (pp.122140). Oxford-New York: Routledge.

Michelini, F. \& Köchy, K. (Eds.). (2020). Jakob von Uexküll and Philosophy. Oxford-New York: Routledge.

Mildenberger, F. (2007). Umwelt als Vision. Leben und Werk Jakob von Uexkülls (1866-1944). Stuttgart: Steiner.

Moreno Mancipe, D. F. (2020). La intencionalidad como impulso formador: Notas para una lectura fenomenológica del origen conjunto del organismo y 
su mundo circundante. Universitas Philosophica, 37(75), 45-72. https://doi. org/10.11144/Javeriana.uph37-75.imff

Moyle, T. (2020). Animal Behavior and the Passage to Culture: Merleau-Ponty's Remarks on Uexküll. F. Michelini \& K. Köchy (Eds.), Jakob von Uexküll and Philosophy (pp.141-157). Oxford-New York: Routledge.

Muñoz Pérez, E. (2015). El aporte de Jakob von Uexküll a Los conceptos fundamentales de la metafísica. Mundo, finitud, soledad (1929-1930) de Martin Heidegger. Diánoia, 60(75), 85-103.

Olivé Pérez, A. (2018). La perspectiva filosófica y biológica de la noción orteguiana de "vida" (Tesis doctoral inédita). Universidad de Barcelona, Barcelona, España.

Ortega y Gasset, J. (1965). Prólogos a la Biblioteca de Ideas del Siglo XX. En: Meditaciones de la técnica (pp. 132-141). Madrid: Espasa-Calpe.

Ostachuk, A. (2013). El Umwelt de Uexküll y Merleau-Ponty. Ludus Vitalis, $21(39), 45-65$.

Scheler, M. (2003). El puesto del hombre en el cosmos. (Trad. J. Gaos). Buenos Aires: Losada.

Sebeok, T. (2001). Biosemiotics: Its Roots, Proliferation, and Prospects. Semiotica, 134(1/4), pp. 61-78. https://doi.org/10.1515/semi.2001.014

Sloterdijk, P. (2005). Atmospheric Politics. En: B. Latour \& P. Weibel (Eds.), Making Things Public: Atmospheres of Democracy (pp. 944-955). KarlsruheCambridge: MIT Press-ZKM.

Stella, M. \& Kleisner, K. (2010). Uexküllian Umwelt as Science and as Ideology: The Light and the Dark Side of a Concept. Theory in Biosciences, 129(1), 39-51. https://doi.org/10.1007/s12064-010-0081-0

Stjernfelt, F. (2011). Simple Animals and Complex Biology: Von Uexküll's TwoFold Influence on Cassirer's Philosophy. Synthese, 179(1), 169-186. https:// doi.org/10.1007/s11229-009-9634-5

Tønnessen, M., Maran, T., \& Sharov, A. (2018). Phenomenology and Biosemiotics. Biosemiotics, 11, 323-330. https://doi.org/10.1007/s12304-0189345-8

von Uexküll, J. J. (1909). Umwelt und Innenwelt der Tiere. Berlin: Springer. von Uexküll, J. J. (1910). Die Umwelt. Die neue Rundschau, 21, 638-649. 
von Uexküll, J. J. (1913). Bausteine zu einer biologischen Weltanschauung. Gesammelte Aufsätze. München: F. Bruckmann A-G.

von Uexküll, J. J. (1920a). Biologische Briefe an eine Dame. Berlín: Verlag von Gebrüder Paetel.

von Uexküll, J. J. (1920b). Theoretische Biologie. Berlín: Verlag von Gebrüder Paetel.

von Uexküll, J. J. (1924). La biología de la ostra jacobea. Revista de Occidente [tomo III], 9, 297-331.

von Uexküll, J. J. (1926). Theoretical Biology. (Trad. D. L. MacKinnon). New

York: Harcourt, Brace \& Co.

von Uexküll, J. J. (1930). Die Lebenslehre. Potsdam: Müller und Kiepenheuer Verlag.

von Uexküll, J. J. \& Kriszat, G. (1934). Streifzüge durch die Umwelten von Tieren und Menschen. Berlin: J. Springer.

von Uexküll, J. J. (1937). Die neue Umweltlehre: Ein Bindeglied zwischen

Natur und Kulturwissenschaften. Die Erziehung, 13(5), 185-199. von Uexküll, J. J. (1940). Bedeutungslehre. Leipzig: Verlag von J. A. Barth. von Uexküll, J. J. (1942). Meditaciones biológicas. La teoría de la significación.

(Trad. J. M. Sacristán). Madrid: Revista de Occidente.

von Uexküll, J. J. (1944). Teoría de la vida. Madrid: Summa.

von Uexküll, J. J. (1951). Ideas para una concepción biológica del mundo. (Trad.

R. M. Tenreiro). Buenos Aires: Espasa-Calpe.

von Uexküll, J.J. (2001). The New Concept of Umwelt: A Link Between Science and the Humanities. Semiotica, 134(1/4), pp. 111-123.

von Uexküll, J. J. (2014). Cartas biológicas a una dama. (Trad. T. Bartoletti \& L. C. Adduci). Buenos Aires: Cactus.

von Uexküll, J. J. (2016). Andanzas por los mundos circundantes de los animales y los hombres. (Trad. M. Guntín). Buenos Aires: Cactus.

von Uexküll, T. (2004). A teoria da Umwelt de Jakob von Uexküll. Galáxia. Revista do Programa de Pós-Graduação em Comunicação e Semiótica, 7, $19-48$. von Utekhin, I. (2001). Spanish Echoes of Jakob von Uexküll's Thought. Semiotica, 134(1/4), 635-642. https://doi.org/10.1515/semi.2001.046 